\title{
Compressed supersymmetry and natural neutralino dark matter from top squark-mediated annihilation to top quarks
}

\author{
Stephen P. Martin \\ Physics Department, Northern Illinois University, DeKalb IL 60115 USA \\ and Fermi National Accelerator Laboratory, PO Box 500, Batavia IL 60510
}

The parameters of the Minimal Supersymmetric Standard Model appear to require uncomfortably precise adjustment in order to reconcile the electroweak symmetry breaking scale with the lower mass limits on a neutral Higgs scalar boson. This problem can be significantly ameliorated in models with a running gluino mass parameter that is smaller than the wino mass near the scale of unification of gauge couplings. A "compressed" superpartner mass spectrum results; compared to models with unified gaugino masses, the ratios of the squark and gluino masses to the lightest superpartner mass are reduced. I argue that in this scenario the annihilation of bino-like neutralino pairs to top-antitop quark pairs through top squark exchange can most naturally play the crucial role in ensuring that the thermal relic dark matter density is not too large, with only a small role played by coannihilations. The lightest superpartner mass must then exceed the top quark mass, and the lighter top squark cannot decay to a top quark. These conditions have important implications for collider searches.

\section{Contents}

I. Introduction

II. Compressed supersymmetry

III. Dark matter density and pair annihilation to top quarks

IV. Implications for colliders

V. Outlook

References 


\section{INTRODUCTION}

Softly-broken supersymmetry is a leading candidate to explain the hierarchy of the Planck mass scale and other high-energy scales to the electroweak symmetry breaking mass scale [1]. In extensions of the Standard Model with a fundamental Higgs scalar, obtaining this hierarchy would seem to require tuning of the Higgs squared mass parameter to about one part in $10^{32}$. The Minimal Supersymmetric Standard Model (MSSM) [2] solves this problem by introducing superpartners with masses near the electroweak scale. In addition, with the assumption of $R$-parity conservation, the most dangerous (renormalizable) contributions to proton decay are eliminated, and the lightest supersymmetric particle (LSP) can serve [3]-[7] as the cold dark matter required by cosmology [8]-[10].

However, the fact that the CERN LEP $e^{+} e^{-}$collider did not discover a Standard Model-like light neutral Higgs scalar boson, placing a limit $M_{h^{0}}>114 \mathrm{GeV}$ [11], has put some tension on the allowed parameter space in the MSSM. This is because $M_{h^{0}}$ is bounded above at tree level by $m_{Z}$, and radiative corrections depend on the superpartner masses, which we assume cannot be too large without reintroducing the hierarchy problem. Including the largest radiative corrections at one-loop order ${ }^{\dagger}$ gives:

$$
\begin{array}{r}
M_{h^{0}}^{2}=m_{Z}^{2} \cos ^{2}(2 \beta)+\frac{3}{4 \pi^{2}} \sin ^{2} \beta y_{t}^{2}\left[m_{t}^{2} \ln \left(m_{\tilde{t}_{1}} m_{\tilde{t}_{2}} / m_{t}^{2}\right)+c_{\tilde{t}}^{2} s_{\tilde{t}}^{2}\left(m_{\tilde{t}_{2}}^{2}-m_{\tilde{t}_{1}}^{2}\right) \ln \left(m_{\tilde{t}_{2}}^{2} / m_{\tilde{t}_{1}}^{2}\right)\right. \\
\left.+c_{\tilde{t}}^{4} s_{\tilde{t}}^{4}\left\{\left(m_{\tilde{t}_{2}}^{2}-m_{\tilde{t}_{1}}^{2}\right)^{2}-\frac{1}{2}\left(m_{\tilde{t}_{2}}^{4}-m_{\tilde{t}_{1}}^{4}\right) \ln \left(m_{\tilde{t}_{2}}^{2} / m_{\tilde{t}_{1}}^{2}\right)\right\} / m_{t}^{2}\right] .
\end{array}
$$

where $c_{\tilde{t}}$ and $s_{\tilde{t}}$ are the cosine and sine of a top-squark mixing angle, $m_{\tilde{t}_{1,2}}$ are the top-squark mass eigenvalues, $y_{t}$ and $m_{t}$ are the top-quark Yukawa coupling and mass, and $\tan \beta=v_{u} / v_{d}$ is the ratio of Higgs vacuum expectation values, and for simplicity the Higgs sector is treated in a decoupling approximation with $h^{0}$ much lighter than the other Higgs bosons $A^{0}, H^{0}, H^{ \pm}$. (In this paper, I follow the notations and conventions of [2].) In order to evade the LEP bound, it is clearly helpful to have $m_{t}$ as large as possible, but the experimental central value [12] has fallen recently. It is also required that $\tan \beta$ is not too small. For fixed values of the superpartner masses, it follows that an upper bound within the approximation of eq. (1.1) is

$$
M_{h^{0}}^{2}<m_{Z}^{2} \cos ^{2}(2 \beta)+\frac{3}{4 \pi^{2}} \sin ^{2} \beta y_{t}^{2} m_{t}^{2}\left[\ln \left(m_{\tilde{t}_{2}}^{2} / m_{t}^{2}\right)+3\right]
$$

in the case that the top-squark mixing is adjusted to have the maximum positive impact on $M_{h^{0}}$. In specific model frameworks without carefully adjusted top-squark mixing it is typically found that this bound is not close to saturated, so while a non-zero top-squark mixing is quite useful for satisfying the LEP bounds for a Standard Model-like lightest Higgs scalar, it is also usually necessary for $m_{\tilde{t}_{2}}^{2} / m_{t}^{2}$ to be fairly large.

This is to be contrasted with the condition for electroweak symmetry breaking, which for $\tan \beta$

\footnotetext{
$\dagger$ This approximation is subject to significant further corrections, which are not necessary for the present argument.
} 
not too small takes the form:

$$
m_{Z}^{2}=-2\left(|\mu|^{2}+m_{H_{u}}^{2}\right)-\frac{1}{v_{u}} \frac{\partial}{\partial v_{u}} \Delta V+\mathcal{O}\left(1 / \tan ^{2} \beta\right) .
$$

Here $\Delta V$ is the radiative part of the effective potential with $v_{u}$ treated as a real variable in the differentiation, $\mu$ is the supersymmetry-preserving Higgs mass parameter, and $m_{H_{u}}^{2}$ is the soft supersymmetry breaking mass term for the Higgs field that couples to the top quark, which must be negative near the electroweak scale. The "supersymmetric little hierarchy problem" is that if supersymmetry breaking parameters are large enough to make $M_{h^{0}}$ exceed the LEP bounds, then a tuning at the several percent-level (or worse) might seem to be needed in eq. (1.3), so that $|\mu|^{2}$ and $-m_{H_{u}}^{2}$ nearly cancel. It has been argued that the level of fine tuning required can be quantified with various measures, but it is my view that any such metrics are inherently and unavoidably subjective, so they will not be used here. Although the little hierarchy problem does not admit of rigorous judgments, it can and does cause discomfort and doubt regarding the likelihood of finding supersymmetric particles in present and future collider searches.

There is no sense in which $|\mu|$ is naturally large, in fact it could naturally be 0 even in the presence of arbitrary supersymmetry breaking if it were not for experimental constraints. The radiative effective potential contribution to eq. (1.3) is not negligible, but since it is loop-suppressed, it does not imply a drastic fine tuning. Therefore, the supersymmetric little hierarchy problem, if indeed there is one, is implied by the fact that $\left|m_{H_{u}}^{2}\right|$ might be expected to be much larger than $m_{Z}^{2}$ in models with heavy top squarks. This indeed occurs in popular models with few parameters with universal soft supersymmetry breaking terms imposed near the scale of apparent gauge coupling unification (the GUT scale), hereafter referred to as mSUGRA. However, it has long been appreciated that this connection is modified or lost in more general models of supersymmetry breaking. In section II I will review the arguments that suggest that the little hierarchy problem is ameliorated in particular by models that predict a smaller gluino mass than in unified models.

A further source of tension on the parameter of the MSSM is provided by the opportunity of the explaining the cold dark matter by the thermal relic density of a neutralino LSP $\left(\tilde{N}_{1}\right)$. Roughly, the annihilation rate for neutralinos decreases with increasing supersymmetry breaking masses in the absence of special mechanisms dependent on particular mass ratios. If the LSP is bino-like, as predicted by many mSUGRA models, then the predicted thermal relic abundance is often found to be too high ${ }^{\ddagger}$ compared to the results of WMAP and other experiments [8]-[10]. The exceptional possibilities have lately been classified qualitatively in four main categories, depending on the mechanism most responsible for reducing the predicted dark matter density to an acceptable level.

First, in the "bulk region" of parameter space, there is a relatively light neutralino LSP, which pair annihilates by the $t$-channel and $u$-channel exchange of sleptons. However, in mSUGRA and similar models, this bulk region often predicts that $M_{h^{0}}$ is too small, or that other states should have been detected at LEP or the Fermilab Tevatron $p \bar{p}$ collider, or gives trouble with other indirect constraints.

\footnotetext{
¥ It is also important that the dark matter need not be neutralinos with a thermal relic density. The LSP might be a gravitino or axino, or something else. Or, if the predicted thermal relic abundance of neutralino dark matter is too low or too high, it can be enhanced or diluted by some non-thermal effect; see for example [13]. However, models that can explain the dark matter without multiplying hypotheses should be accorded special interest.
} 
Second, in the Higgs resonance (or funnel) region, neutralino pairs annihilate through the $s$ channel exchange of the pseudo-scalar Higgs boson $A^{0}$ in an $s$-wave final state. Because the relevant coupling is proportional to $m_{b} \tan \beta$, this usually entails large values of $\tan \beta$ [4]. (There is also the possibility of annihilating near the $h^{0}$ pole [14].)

Third, there is the possibility that the LSP has a significant higgsino component, allowing larger neutralino pair annihilation and co-annihilation with the heavier neutralinos and charginos, to and through weak bosons [15]. This occurs for example in the "focus point" region of parameter space, in which $|\mu|$ is not too large, even if the sfermions are very heavy [16].

A fourth possibility, the "sfermion co-annihilation region" of parameter space [17], is obtained if there is a sfermion (typically a tau slepton [18] in mSUGRA, but possibly a top squark [19]-[23]) that happens to be slightly heavier than the LSP. A significant density of this sfermion will then coexist with the LSP around the freeze-out time, and so annihilations involving the sfermion with itself or with the LSP will further dilute the number of superpartners and so the eventual dark matter density. The co-annihilation region generally requires just the right mass difference between the stau or stop quark and the LSP, and so is often considered to be fine tuned.

If the LSP is mostly higgsino or wino, then the annihilation of superpartners in the early universe is typically too efficient to provide for thermal relic density in agreement with WMAP. However, one can always adjust the higgsino or wino contents of $\tilde{N}_{1}$ to be just right, at the expense of some fine tuning. In recent years, there have been many studies of the properties of neutralino dark matter that follow from abandoning the strict boundary conditions of mSUGRA models to allow non-universal gaugino masses [24]- 39] or scalar masses [40]-[46] at the GUT scale. By increasing the wino or higgsino content of the neutralino, one can increase the cross-section for annihilations and co-annihilations to weak bosons, and those mediated by the $Z$ boson and $h^{0}$ and $A^{0}$ in the $s$-channel.

In section III of this paper, I will study a different possibility with rather distinctive properties, namely the possibility that the LSP is mostly bino-like, but pair annihilates efficiently to top-antitop quark pairs due predominantly to the exchange of light top squarks. In the models discussed in section I (unlike in mSUGRA and similar models) this mechanism turns out to give a thermal relic dark matter density in agreement with the WMAP measurements for a wide range of input parameters, much more than in the stop co-annihilation (or stau co-annihilation) regions to which it is continuously connected. This scenario also has important implications for collider searches at the Fermilab Tevatron, CERN Large Hadron Collider (LHC), and a future linear collider, discussed briefly in section IV] Section V] contains some concluding remarks.

\section{COMPRESSED SUPERSYMMETRY}

In this section, I review the argument that a suppression of the gluino mass parameter ameliorates the little hierarchy problem in supersymmetry. (This has been observed in various papers; a particularly clear and early explanation was given in ref. [47].)

As noted in the Introduction, the issue is essentially to explain why the running parameter $-m_{H_{u}}^{2}$ should be small and positive near the electroweak scale, in the same theory that allows large positive corrections to $M_{h^{0}}^{2}$. The parameter $|\mu|^{2}$, which relies on a different sector of the theory, can then be chosen without too much fine tuning to give the right $m_{Z}^{2}$ in eq. (1.3). In terms 
of the MSSM soft supersymmetry breaking parameters at the apparent GUT scale, one finds:

$$
\begin{aligned}
-m_{H_{u}}^{2}= & 1.92 \hat{M}_{3}^{2}+0.16 \hat{M}_{2} \hat{M}_{3}-0.21 \hat{M}_{2}^{2}-0.33 \hat{M}_{3} \hat{A}_{t}-0.074 \hat{M}_{2} \hat{A}_{t}+0.11 \hat{A}_{t}^{2} \\
& +0.024 \hat{M}_{1} \hat{M}_{3}+0.006 \hat{M}_{1} \hat{M}_{2}-0.006 \hat{M}_{1}^{2}-0.012 \hat{M}_{1} \hat{A}_{t}+0.002 \hat{M}_{3} \hat{A}_{b} \\
& -0.63 \hat{m}_{H_{u}}^{2}+0.36 \hat{m}_{Q_{3}}^{2}+0.28 \hat{m}_{u_{3}}^{2}-0.027 \hat{m}_{H_{d}}^{2}+0.025 \hat{m}_{d_{3}}^{2}-0.026 \hat{m}_{L_{3}}^{2} \\
& +0.026 \hat{m}_{e_{3}}^{2}+0.05 \hat{m}_{Q_{1}}^{2}-0.11 \hat{m}_{u_{1}}^{2}+0.05 \hat{m}_{d_{1}}^{2}-0.05 \hat{m}_{L_{1}}^{2}+0.05 \hat{m}_{e_{1}}^{2}
\end{aligned}
$$

Here, the hats on the parameters on the right-hand side denote that they are inputs at the apparent GUT scale, while $m_{H_{u}}^{2}$ on the left-hand side denotes the result at the renormalization scale $Q=400$ $\mathrm{GeV}$ (where the corrections due to the effective potential are presumed moderate), using $\tan \beta=10$ and the SPS1a' benchmark point [48] values for the Yukawa and gauge couplings and unification scale, and using two-loop renormalization group equations [49]. The input parameters consist of independent gaugino masses $\hat{M}_{1}, \hat{M}_{2}, \hat{M}_{3}$, scalar trilinear coupling parameters $\hat{A}_{t}, \hat{A}_{b}, \hat{A}_{\tau}$, and scalar squared masses for the Higgs bosons, third family sfermions, and first family sfermions (with each second family sfermion assumed degenerate with the first family counterpart having the same quantum numbers). Some contributions with very small coefficients have been omitted from eq. (2.1). The reason for applying boundary conditions at the GUT mass is that the apparent unification of couplings provides some justification that it is meaningful to extrapolate running parameters up to that scale.

In the so-called mSUGRA framework, the input parameters are usually taken to obey the much stronger conditions:

$$
\begin{aligned}
& \hat{M}_{1}=\hat{M}_{2}=\hat{M}_{3}=m_{1 / 2}, \\
& \hat{A}_{t}=\hat{A}_{b}=\hat{A}_{\tau}=A_{0}, \\
& \hat{m}_{\phi}^{2}=m_{0}^{2}
\end{aligned}
$$

for all scalars $\phi=H_{u}, H_{d}, Q_{i}, u_{i}, d_{i}, L_{i}, e_{i}$, with family index $i=1,2,3$. It is then clear that the largest contribution to $-m_{H_{u}}^{2}$ at the weak scale is due to the input gluino mass $\hat{M}_{3}$; furthermore, there is a significant cancellation between the scalar contributions within the mSUGRA framework.

Generalizing the input parameters can provide a relative reduction in $-m_{H_{u}}^{2}$, therefore lowering both the predicted value of $|\mu|^{2}$ and the cancellation needed to obtain the observed value of $m_{Z}$. From consideration of the first five terms on the right-hand side, one learns that small values of $|\mu|$ result from (roughly) $\hat{M}_{3} \approx 0.29 \hat{M}_{2}+0.13 \hat{A}_{t}$ or $\hat{M}_{3} \approx-0.38 \hat{M}_{2}+0.04 \hat{A}_{t}$, provided that $\hat{M}_{1}$, $\hat{A}_{t}, \hat{m}_{H_{u}}^{2}$ etc. are not too large. A complete cancellation is actually not desirable from our present point of view, since a mostly higgsino-like neutralino has too small a thermal relic density, and $M_{h^{0}}$ often comes out too small. There are many types of models already in the literature that can predict a small ratio of $\hat{M}_{3} / \hat{M}_{2}$. The scenario for dark matter to be studied in the next section does not depend crucially on which framework is used, but for concreteness I will review one here.

In the usual mSUGRA framework, one assumes that the gaugino masses are all the same; in $S U(5)$ GUT language this corresponds to a supersymmetry breaking $F$-term in a singlet of $S U(5)$ [or $S O(10)$ ]. More generally, one can consider non-universal gaugino masses arising from an $F$ term $\mathrm{VEV}$ in arbitrary linear combinations of the symmetric product of two adjoint representations of 
the GUT group that contain a Standard Model singlet [50]-[53]. For $S U(5)$ :

$$
(24 \times 24)_{S}=1+24+75+200 .
$$

The resulting gaugino mass terms have the form

$$
\mathcal{L}=-\sum_{R} \frac{\left\langle F_{R}\right\rangle}{2 M_{P}} \sum_{n} c_{R}^{(n)} \lambda_{n} \lambda_{n}+\text { c.c. }
$$

where the coefficients $c_{R}^{(n)}$ (with $n=1,2,3$ for bino, wino, and gluino respectively, and $R=$ $\mathbf{1}+\mathbf{2 4}+\mathbf{7 5}+\mathbf{2 0 0}$ ) are determined by group theory, leading to the parameterization:

$$
\begin{aligned}
& \hat{M}_{1}=m_{1 / 2}\left(1+C_{24}+5 C_{75}+10 C_{200}\right), \\
& \hat{M}_{2}=m_{1 / 2}\left(1+3 C_{24}-3 C_{75}+2 C_{200}\right), \\
& \hat{M}_{3}=m_{1 / 2}\left(1-2 C_{24}-C_{75}+C_{200}\right) .
\end{aligned}
$$

The special case $C_{24}=C_{75}=C_{200}$ recovers the mSUGRA model. In eqs. (2.7)-(2.9), I have assumed that there is at least some $S U(5)$ singlet component to the $F$ term, although this is not strictly necessary. Note that this parameterization is already general enough to fit any observed gaugino mass hierarchy, simply because it contains three linearly independent contributions.

One particularly simple way to achieve a ratio $\hat{M}_{3} / \hat{M}_{2} \sim 1 / 3$ is to choose $C_{24} \sim 0.22$, with $C_{75}=C_{200}=0$. This is the inspiration for the model space studied in the next section, although it cannot be overemphasized that there are many other reasonable ways to achieve such a ratio. One point in favor of this type of model is that in GUT theories like $S U(5)$, there is a chiral superfield in the $\mathbf{2 4}$ (adjoint) representation anyway; once the scalar component acquires a VEV, it is actually unnatural for the $F$ term to not develop a VEV as well. Moreover, this can make the theory consistent with proton decay requirements and help to obtain precise gauge coupling unification [54, 55].

As evidenced by the special case of eq. (2.1), soft supersymmetry breaking mass parameters at the weak scale are substantially driven by the gaugino mass parameters through large logarithmic effects that are summarized in the renormalization group. In mSUGRA models, this effect typically causes squarks and the gluino to be much heavier than the superpartners that do not have $S U(3)_{C}$ interactions, except when $m_{0}$ is very large. In the case of a small ratio $\hat{M}_{3} / \hat{M}_{2}$ motivated by a solution to the little hierarchy problem, however, the resulting mass spectrum will be "compressed" in comparison to mSUGRA, with a smaller ratio of the masses of the heaviest and lightest superpartners. Two aspects of this that will be important in the next section are that it becomes much more likely that the lighter top squark can be the next-to-lightest supersymmetric particle (NLSP), and that the LSP is rather heavy.

It will be assumed here that the trilinear scalar couplings are sizable and negative at the GUT scale (in the convention of [2]). This can be motivated as the effect of strong renormalization group running between the GUT scale and the Planck scale, which would produce both negative scalar trilinear couplings and positive scalar squared masses, and often prefers positive $\mu$ [56] (independently of low-energy $(g-2)_{\mu}$ or $b \rightarrow s \gamma$ considerations), when the running is dominated by 
positive gaugino masses. It is worth noting that in many viable supersymmetric GUT theories, the naive running of the gauge couplings above the unification scale quickly becomes non-perturbative. For example, in the minimal missing partner supersymmetric $S U(5)$ theory [57], the two-loop gauge beta function has a Landau pole, and the three- and four-loop beta functions appear to have strongly-coupled UV-stable fixed points [58]. While the breakdown of perturbation theory renders such calculations untrustworthy in detail, this suggests that gaugino mass dominance could eliminate the problem of supersymmetric flavor-violation, while giving essentially arbitrary flavorpreserving soft supersymmetry breaking terms at the GUT scale. As a simplistic assumption made only for convenience, the scalar trilinear couplings $\hat{A}_{t}, \hat{A}_{b}, \hat{A}_{\tau}$ will be taken to be unified in the models below; the parameter $\hat{A}_{t}$ has the most direct importance in most cases.

Likewise I will assume, as a matter of convenience, that at the GUT scale all scalars have a common squared mass $m_{0}^{2}$ as in mSUGRA. While it is clearly worthwhile to consider scalar mass non-universality at the GUT scale, I expect that the results obtained here will be realized at least qualitatively in a variety of different schemes without universal scalar squared masses imposed at the GUT scale.

\section{DARK MATTER DENSITY AND PAIR ANNIHILATION TO TOP QUARKS}

In order to explain a large value of $M_{h^{0}}$ in models of compressed supersymmetry, it is favored that the mass spectrum is compressed up, rather than down. This means that the LSP will have to be heavier than usually found in the mSUGRA "bulk" region for dark matter.

It has been suggested in models of this type with small $\left|M_{3} / M_{2}\right|$ that the thermal relic abundance of dark matter can be explained by an enhanced Higgsino component of $\tilde{N}_{1}$, leading to enhanced annihilations $\tilde{N}_{1} \tilde{N}_{1} \rightarrow W^{+} W^{-}$or $Z Z[28,35]$, or by $s$-channel annihilation through the pseudoscalar Higgs $A^{0}$ near resonance [28, 31, 33], or by co-annihilations with heavier neutralinos and charginos [31, 35], or by $s$-channel annihilations to $t \bar{t}$ through the $Z$ boson [28, 33].

In this paper, I will consider instead the case that the thermal relic density is suppressed primarily by $\tilde{N}_{1}$ pair annihilation to top quark-antiquark pairs, mediated mostly by top-squark exchange. As mentioned in the previous section, it is not difficult in compressed supersymmetric models to obtain a top squark NLSP. This is in contradistinction to mSUGRA and similar models, where achieving the required suppression in $\Omega_{\mathrm{DM}} h^{2}$ from top-squark exchange requires that $\left|A_{0}\right|$ is very large in absolute terms and must be rather finely adjusted so that $\tilde{t}_{1}$ is not much heavier than $\tilde{N}_{1}$ (see for example refs. [20, 21, 23]). In the points in mSUGRA parameter space where this can occur, $\tilde{t}_{1} \tilde{t}_{1}$ and $\tilde{N}_{1} \tilde{t}_{1}$ co-annihilations are also generally very important (unlike here). Compressed supersymmetry models with small ${ }^{\dagger}\left|M_{3} / M_{2}\right|$ at the GUT scale have the crucial distinction that achieving comparable $m_{\tilde{t}_{1}}$ and $m_{\tilde{N}_{1}}$ is much easier, and requires smaller values of $\left|A_{t}\right|$ in absolute terms, and admits a wider range of $A_{t}$.

The tree-level Feynman diagrams that contribute to the process $\tilde{N}_{1} \tilde{N}_{1} \rightarrow t \bar{t}$ are shown in Figure 1, In order to obtain $\Omega_{\mathrm{DM}} h^{2}$ compatible with WMAP by this mechanism (without undue

\footnotetext{
${ }^{\dagger}$ Hats will be omitted from GUT scale input parameters throughout this section.
} 

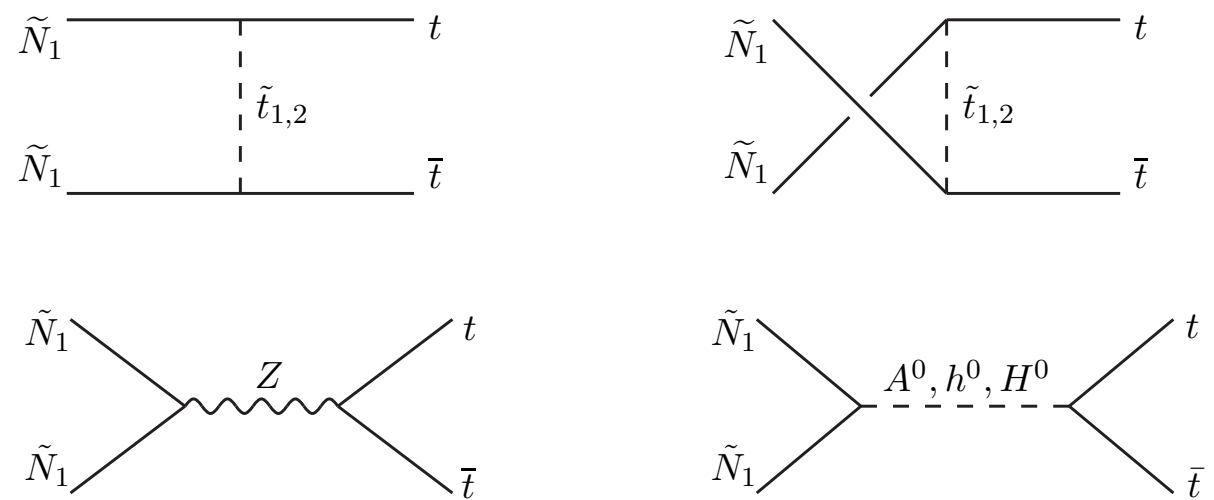

FIG. 1: Contributions to the annihilation of neutralino dark matter LSP pairs into top quark-antiquark pairs, from top squark, $Z$ boson, and Higgs boson exchange.

fine tuning), it is necessary that:

$$
\begin{aligned}
& m_{t}<m_{\tilde{N}_{1}} \lesssim m_{t}+100 \mathrm{GeV} \\
& m_{\tilde{N}_{1}}+25 \mathrm{GeV} \lesssim m_{\tilde{t}_{1}} \lesssim m_{\tilde{N}_{1}}+100 \mathrm{GeV}
\end{aligned}
$$

The first inequality in eq. (3.2) is the approximate requirement that the relic density not be suppressed too much by top-squark co-annihilations. The upper bounds here are also necessarily fuzzy, because of the connection to the thin co-annihilation region. For models satisfying these criteria, the top-squark exchange is most important for bino-like $\tilde{N}_{1}$ and $\tilde{t}_{1}$ with a high $\tilde{t}_{R}$ component. As one increases the small Higgsino component of $\tilde{N}_{1}$ (by lowering $|\mu|$ ), the contribution from the $\tilde{t}_{1}$ exchange diagrams becomes enhanced, due to the top Yukawa coupling. In the models to be considered below, the $s$-channel $Z$ exchange diagram is subdominant but not negligible; using the analytic formulas provided in [4], one can show that the most important effect is a significant destructive interference with the dominant top-squark exchange diagram amplitude.

For a more detailed study, I have used the program micrOMEGAs 2.0.1 [6] to evaluate the thermal relic abundance of dark matter for supersymmetric models generated using SOFTSUSY 2.0.11 [59] (and checked for approximate agreement with SuSpect [60]). In the following, I consider a rather conservative thermal relic density constraint:

$$
0.09<\Omega_{\mathrm{DM}} h^{2}<0.13
$$

and impose a Higgs mass constraint:

$$
M_{h^{0}}>113 \mathrm{GeV}
$$

This is slightly lower than the LEP bound for Standard Model-like Higgs scalars, justified by the significant uncertainties involved in the theoretical Higgs mass calculation. In addition, I adopt the slightly optimistic value of $m_{t}=175 \mathrm{GeV}$ rather than the somewhat lower latest combined central value $m_{t}=171.4 \pm 1.8$ (syst.) \pm 1.2 (stat.) $\mathrm{GeV}$ from the Tevatron [12]. In each model, I require that the LSP is a neutralino. Then all limits on supersymmetric particles from LEP 
turn out to be automatically satisfied. No constraint from the anomalous magnetic moment of the muon is applied, since for all models considered here, the predicted value is actually closer to the experimental central value(s) from the BNL E821 experiment [61] than the Standard Model prediction is (but not by a very statistically significant amount). I do not impose any bound coming from $b \rightarrow s \gamma$, since the measurement can be easily accommodated [62] by introducing some extra small GUT-scale flavor violation in the supersymmetry-breaking parameters, in a way that would not affect the rest of the model in any appreciable way.

Results for a typical two-parameter model space are shown in Figure 2. Here I consider models with boundary conditions at the GUT scale:

$$
1.5 M_{1}=M_{2}=3 M_{3},
$$

with $M_{1}$ and $m_{0}$ allowed to vary independently, $\tan \beta=10$ and $\mu>0$, and two values for the ratio $A_{0} / M_{1}=-0.75$ (outlined region) and $A_{0} / M_{1}=-1$ (shaded region). The allowed regions are cut off on the lower left by the Higgs mass bound constraint eq. (3.4). The upward bulges in the regions are the places where top-squark exchange plays the dominant role in $\tilde{N}_{1} \tilde{N}_{1} \rightarrow t \bar{t}$, which in turn is the most important annihilation process for the dark matter. Typically, in the bulge regions $\tilde{N}_{1} \tilde{N}_{1} \rightarrow t \bar{t}$ accounts for $70 \%$ to $90 \%$ of the contributions to $1 / \Omega_{\mathrm{DM}} h^{2}$. Each of these regions is continuously connected to much narrower co-annihilation regions on either side. For $A_{0} / M_{1}=-1$, stop co-annihilation is the dominant effect in these thin strips, but for $A_{0} / M_{1}=-0.75$, stau co-annihilations are also important there.

For smaller values of $-A_{0} / M_{1}$, not shown here, the $\tilde{N}_{1} \tilde{N}_{1} \rightarrow t \bar{t}$ bulge region still exists, but continuously connects instead to a thin stau co-annihilation strip.

As can be seen from Figure 2, the smaller $-A_{0}$ case requires a larger mass difference $m_{\tilde{t}_{1}}-m_{\tilde{N}_{1}}$ in the allowed bulge region. This can be traced to the fact that $\mu$ decreases as $-A_{0}$ decreases [see eqs. (1.3) and (2.1)], slightly enhancing the small Higgsino component of the LSP, which substantially increases the top-squark exchange amplitude contributions to the annihilation cross section as mentioned above.

The $s$-channel Higgs scalar annihilation diagrams shown in Figure1play only a small role in the LSP pair annihilation in these models. This is because in all cases $m_{\tilde{N}_{1}}$ is well below the resonance point $m_{A^{0}} / 2 \approx m_{H^{0}} / 2$, and well above the resonance point $m_{h^{0}} / 2$.

Also shown in Figure 2 are the critical lines that govern the decay of $\tilde{t}_{1}$. The lowest dashed line indicates the region allowed by the requirement that $\tilde{N}_{1}$ is the LSP. In all cases, the decay $\tilde{t}_{1} \rightarrow t \tilde{N}_{1}$ is kinematically closed, as indicated by the upper dashed line. Above the middle dashed line, the decay

$$
\tilde{t}_{1} \rightarrow W b \tilde{N}_{1}
$$

is kinematically open, and should dominate. Below that line, the flavor-violating 2-body decay

$$
\tilde{t}_{1} \rightarrow c \tilde{N}_{1}
$$

is expected to win [63] over the four-body decays $\tilde{t}_{1} \rightarrow q \bar{q}^{\prime} b \tilde{N}_{1}$ and $\tilde{t}_{1} \rightarrow \ell \nu b \tilde{N}_{1}$. The charginos $\left(\tilde{C}_{i}\right)$ and sleptons are heavier in the models shown, so they cannot appear in $\tilde{t}_{1}$ decays. 


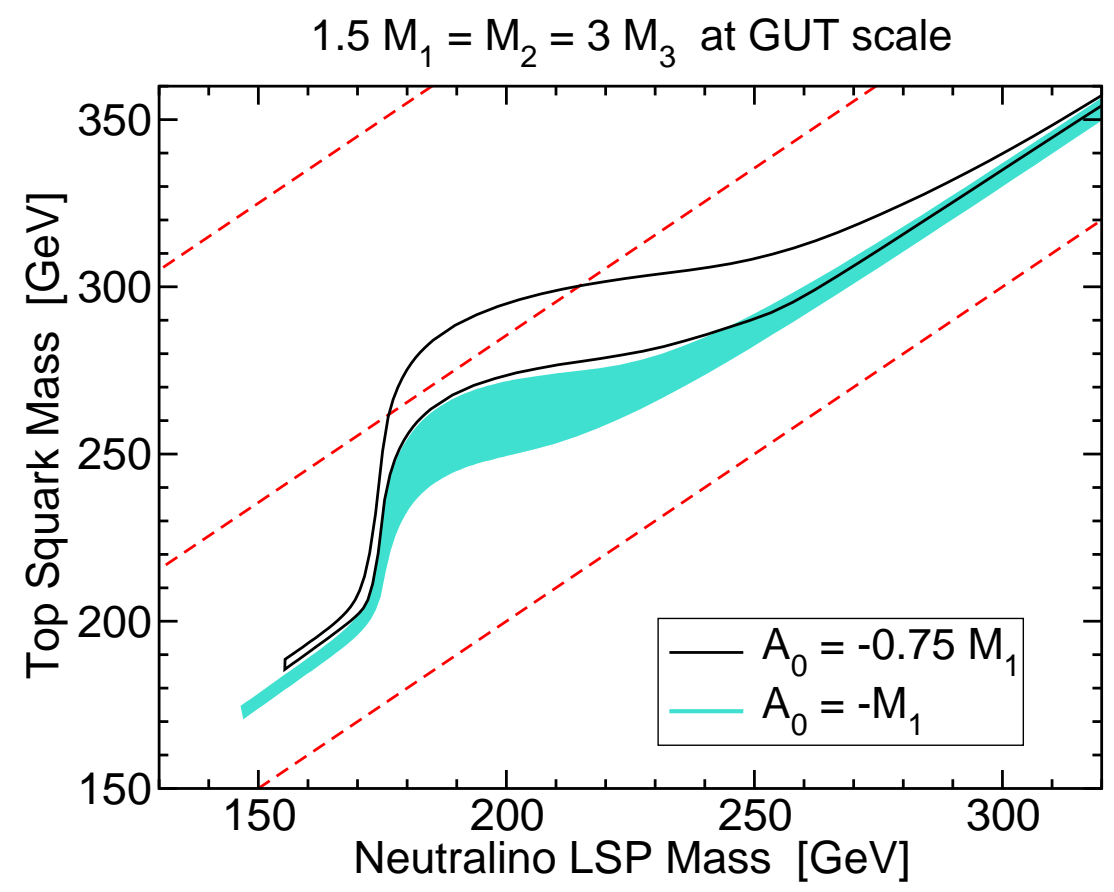

FIG. 2: Allowed regions in the $m_{\tilde{N}_{1}}, m_{\tilde{t}_{1}}$ plane that predict a thermal relic abundance of neutralino LSP dark matter $0.09<\Omega_{\mathrm{DM}} h^{2}<0.13$ and satisfy other constraints given in the text. The GUT-scale parameters satisfy $1.5 M_{1}=M_{2}=3 M_{3}$, with variable $m_{0}$, and $A_{0}=-0.75 M_{1}$ (region outlined in black) and $A_{0}=-M_{1}$ (shaded region). Also, $\tan \beta=10$ and $\mu>0$. The lowest dashed line is $m_{\tilde{t}_{1}}=m_{\tilde{N}_{1}}$. Below the upper dashed line, the decay $\tilde{t}_{1} \rightarrow t \tilde{N}_{1}$ is forbidden. Below the middle dashed line, the three-body decay $\tilde{t}_{1} \rightarrow W b \tilde{N}_{1}$ is also forbidden.

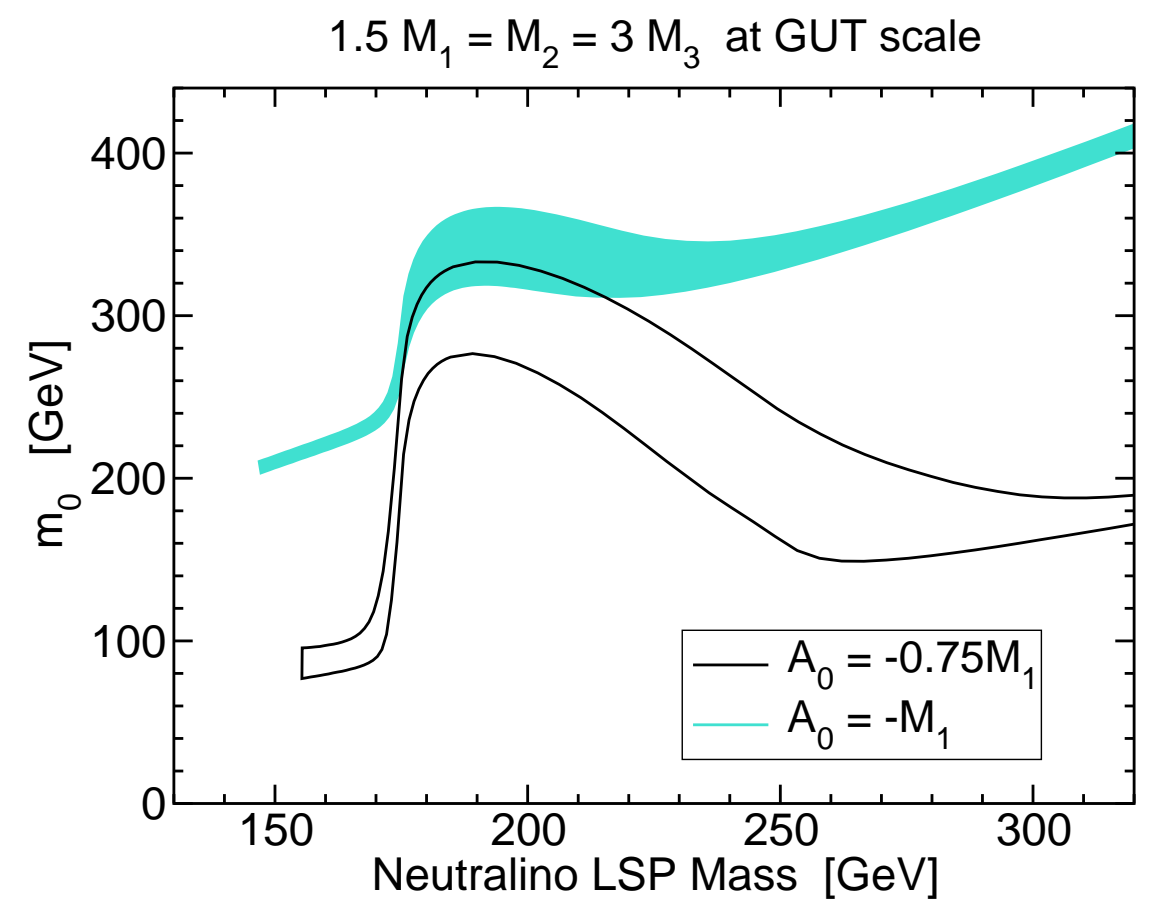

FIG. 3: The allowed regions depicted in Figure 1 arise from the values of $m_{0}$ shown here. 


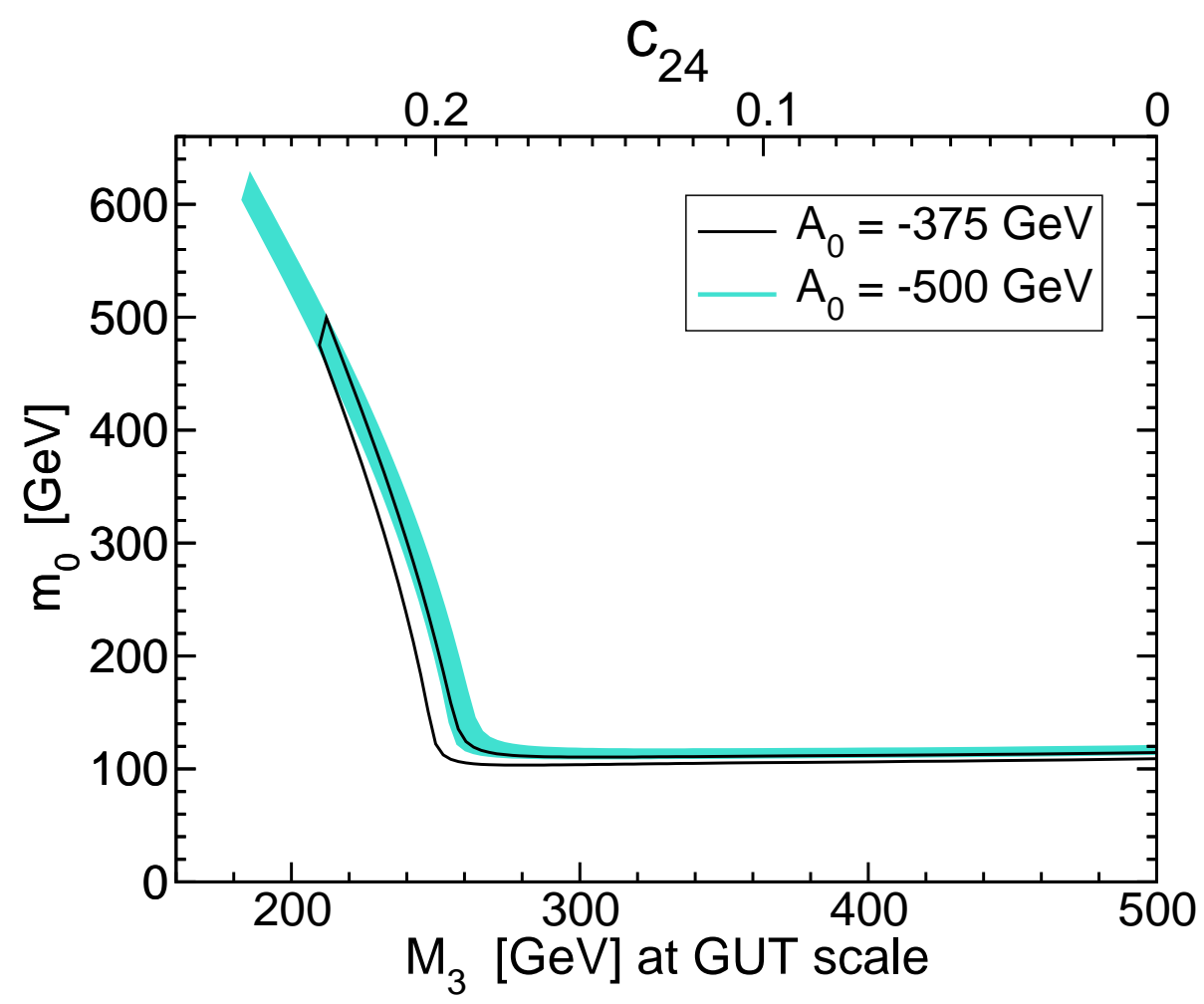

FIG. 4: Allowed regions that predict a thermal relic abundance of neutralino LSP dark matter $0.09<$ $\Omega_{\mathrm{DM}} h^{2}<0.13$ and satisfy other constraints given in the text. At the GUT scale, the bino mass parameter $M_{1}=500 \mathrm{GeV}$ is fixed, and the wino and gluino mass parameters vary while obeying eqs. (2.7)-(2.9) with $C_{75}=C_{200}=0$. The horizontal direction is parameterized by $M_{3}$ at the GUT scale (lower horizontal axis) or equivalently by $C_{24}$ (upper horizontal axis). The vertical axis is the common scalar mass $m_{0}$. The region outlined in black has $A_{0}=-375 \mathrm{GeV}$ and the shaded region has $A_{0}=-500 \mathrm{GeV}$, with $\tan \beta=10$ and $\mu>0$ in each case. The very thin, nearly horizontal regions with $m_{0}$ near $110 \mathrm{GeV}$ feature co-annihilation of sleptons and the LSP. In the thicker sloping areas on the left, the dominant contribution to $1 / \Omega_{\mathrm{DM}} h^{2}$ is $\tilde{N}_{1} \tilde{N}_{1} \rightarrow t \bar{t}$, mostly due to the $\tilde{t}_{1}$ exchange diagram amplitudes.

The relative thickness of the allowed regions cannot be regarded as a direct measure of finetuning, even subjectively. In fact, a perfectly accurate determination of $\Omega_{\mathrm{DM}} h^{2}$ would make the allowed regions arbitrarily thin, up to model assumption and theoretical errors and input parameter uncertainties..$^{\ddagger}$ (The Planck satellite mission experiment 64 should indeed significantly improve the determination.) However, it seems clear that the observed dark matter density is more naturally accommodated in the $\tilde{N}_{1} \tilde{N}_{1} \rightarrow t \bar{t}$ bulge regions, since a larger range of $m_{0}$ values (for each fixed $M_{1}$ ) lie within the range specified by eq. (3.3). This is illustrated for the same models in Figure 3 , Notably, all of the soft supersymmetry breaking parameters are less than the gaugino masses $M_{1}$ and $M_{2}$; this includes the holomorphic term $b=B \mu$, which is of order $(250 \mathrm{GeV})^{2}$ in the bulge region of these models.

Another handle on the $\tilde{N}_{1} \tilde{N}_{1} \rightarrow t \bar{t}$ annihilation scenario is provided by Figure 4 , which shows dark matter allowed regions in the plane of $m_{0}$ vs. $M_{3}$, for models with fixed $M_{1}=500 \mathrm{GeV}$ at the

\footnotetext{
$\ddagger$ Moreover, one could regard the entire parameter space between the indicated regions and the $m_{\tilde{t}_{1}}=m_{\tilde{N}_{1}}$ line as allowed, in the sense that the thermal relic abundance would be less than or equal to the observed value. Then something else, for example axions, would make up the remaining dark matter.
} 


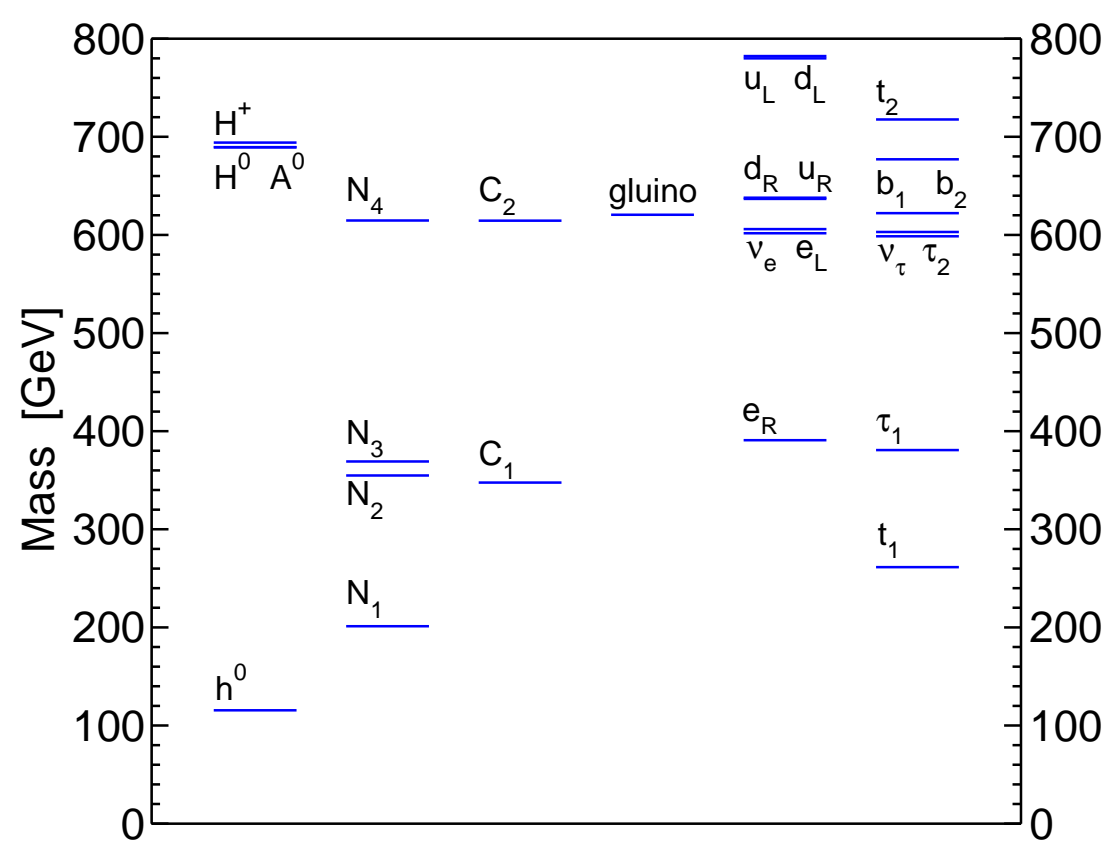

FIG. 5: A typical sample "compressed" Higgs and superpartner mass spectrum with $\Omega_{\mathrm{DM}} h^{2}=0.11$ brought about by $\tilde{N}_{1} \tilde{N}_{1} \rightarrow t \bar{t}$ through $\tilde{t}_{1}$ exchange. The GUT scale parameters of the model are $M_{1,2,3}=500,750,250, A_{0}=-500$, and $m_{0}=342 \mathrm{GeV}$, with $\tan \beta=10$ and $\mu>0$ at the weak scale. The ratio of the largest superpartner mass to the smallest is less than 4 . An unfortunate feature, quite common to this scenario for dark matter, is that no visible superpartners would be within reach of a linear collider with $\sqrt{s}=500 \mathrm{GeV}$.

GUT scale (so that the LSP mass is approximately $200 \mathrm{GeV}$ ) and obeying the boundary condition of eqs. (2.7)-(2.9) with $C_{24}$ varying and $C_{75}=C_{200}=0$. I again require $\mu>0$ and $\tan \beta=10$, and the allowed regions are shown for $A_{0}=-M_{1}$ and $A_{0}=-0.75 M_{1}$. The thin horizontal regions achieve the observed dark matter density by co-annihilations of sleptons and the LSP; as is wellknown, this requires a rather precise adjustment of the slepton squared masses. For $C_{24} \gtrsim 0.19$, or equivalently $M_{3} \lesssim 260 \mathrm{GeV}$, the $\tilde{N}_{1} \tilde{N}_{1} \rightarrow t \bar{t}$ annihilation scenario takes over, leading to the thicker, sloping allowed regions. They are cut off on the left by the imposed Higgs mass constraint eq. (3.4).

The distinctive features of the $\tilde{N}_{1} \tilde{N}_{1} \rightarrow t \bar{t}$ annihilation scenario for dark matter in compressed supersymmetry are illustrated in the superpartner spectrum for a typical model point shown in Figure 5, with $M_{1}=500 \mathrm{GeV}$ and $m_{0}=342 \mathrm{GeV}$ in order to give $\Omega_{\mathrm{DM}} h^{2}=0.11$. In this model, $\tilde{N}_{1} \tilde{N}_{1} \rightarrow t \bar{t}$ contributes about $89 \%$ to $1 / \Omega_{\mathrm{DM}} h^{2}$. The amplitude from $\tilde{t}_{1}$ exchange is largest, with an amplitude from $Z$ exchange about 0.3 times as big in the velocity-independent part of the ${ }^{1} S_{0}$ channel, with destructive interference. The superpartner mass spectrum shows compression compared to mSUGRA models, with the ratio of masses of the largest superpartners (nearly degenerate $\tilde{u}_{L}, \tilde{c}_{L}$ and $\tilde{d}_{L}, \tilde{s}_{L}$ ) to the LSP being less than 4 , with all superpartners between $200 \mathrm{GeV}$ and $800 \mathrm{GeV}$. The NSLP is $\tilde{t}_{1}$. The lightest chargino $\tilde{C}_{1}$ and the neutralinos $\tilde{N}_{2}$ and $\tilde{N}_{3}$ are higgsino-like; this is a consequence of $\mu$ being not too large as discussed in section II. Another consequence of the choice of a relatively large wino mass to ameliorate the little hierarchy problem is that the wino-like states $\tilde{N}_{4}$ and $\tilde{C}_{2}$ are comparatively heavy, just below the gluino mass, and there is a wide split between left-handed squarks and sleptons and their right-handed counterparts. 


\section{IMPLICATIONS FOR COLLIDERS}

In this section I will make some brief remarks on the implications of the scenario outlined above for collider searches. Figure 5 shows a typical model of this type, with the characteristic feature that the decay $\tilde{t}_{1} \rightarrow c \tilde{N}_{1}$ has a $100 \%$ branching fraction. For this section, I will use this as a benchmark, with the important caveat that search strategies will be qualitatively different if the decay $\tilde{t}_{1} \rightarrow W b \tilde{N}_{1}$ is open. Other notable decays, with approximate branching fractions computed by ISAJET 7.74 [65], are:

$$
\tilde{g} \rightarrow \begin{cases}t \tilde{t}_{1}^{*} & (\sim 50 \%) \\ \bar{t} \tilde{t}_{1} & (\sim 50 \%)\end{cases}
$$

for the gluino, and

$$
\begin{aligned}
& \tilde{N}_{2} \rightarrow \begin{cases}\tilde{N}_{1} h & (\sim 90 \%) \\
\tilde{N}_{1} Z & (\sim 10 \%)\end{cases} \\
& \tilde{N}_{3} \rightarrow \tilde{N}_{1} Z \quad(\sim 97 \%) \\
& \tilde{C}_{1} \rightarrow \tilde{t}_{1} b \quad(\sim 95 \%)
\end{aligned}
$$

for the Higgsino-like neutralinos and charginos. The wino-like neutralino and chargino $\tilde{N}_{4}$ and $\tilde{C}_{2}$ are so heavy in this class of models that they are unlikely to be directly produced in great numbers at any foreseeable colliders, but may appear (with small branching fractions) in decays of left-handed squarks. This scarcity of winos is different from the expectation in many mSUGRA models, for example. The left-handed squarks of the first two families decay predominantly through the gluino, while the right-handed squarks decay mostly directly to the LSP:

$$
\begin{aligned}
& \tilde{q}_{L} \rightarrow \begin{cases}q \tilde{g} & (\sim 78 \%) \\
q^{\prime} \tilde{C}_{2} & (\sim 11 \%)\end{cases} \\
& \tilde{q}_{R} \rightarrow q \tilde{N}_{1} \quad(\sim 90 \%)
\end{aligned}
$$

However, the latter large branching fraction is due here to the very small phase space allowed for decays to the gluino, so while it is a real possibility, it cannot be considered a robust prediction for this class of models in general.

The sleptons decay mostly directly to the bino-like LSP $\tilde{N}_{1}$. Unfortunately, they almost completely decouple from the other superpartner decay chains, so they must be produced directly to be observed. This is clearly a challenge, given their large masses.

Because of the large masses of the entire superpartner spectrum, it will be difficult to probe the scenario outlined here at the Tevatron. The process

$$
p \bar{p} \rightarrow \tilde{t}_{1} \tilde{t}_{1}^{*} \rightarrow c \bar{c} \tilde{N}_{1} \tilde{N}_{1} \rightarrow c \bar{c}+\not \mathbb{E}_{T}
$$


is one of the searches being actively pursued by both the $\mathrm{D} \emptyset$ [66] and CDF 67] collaborations, using heavy flavor tags. However, the sensitivity appears to be well short [68] of that needed to probe most of the region favored by neutralino annihilations to top quarks as the solution for dark matter. The same process without the heavy flavor tag requirement, $p \bar{p} \rightarrow($ acoplanar $j j)+\mathbb{E}_{T}$ [69], may also be interesting. However, in the present case the jets will not have a particularly high transverse momentum compared to the typical situation in mSUGRA benchmark models. The clean trilepton and $\mathbb{E}_{T}$ signal from wino-like $\tilde{C}_{1} \tilde{N}_{2}$ production that is found in mSUGRA is unavailable here. The other superpartners generally are too heavy to be produced in sufficient numbers at the Tevatron with the anticipated integrated luminosity. The Higgs sector consists of a Standard-Model like Higgs boson $h^{0}$ and a heavy isotriplet of Higgs bosons $\left(H^{0}, A^{0}, H^{ \pm}\right)$, so enhanced signals are not expected there.

At the Large Hadron Collider, squark and gluino production will dominate as usual. The latter leads to the signal of two tops and two charm jets, but with $50 \%$ probability for like-sign tops, because of the Majorana nature of the gluino decays:

$$
p p \rightarrow \tilde{g} \tilde{g} \rightarrow\left\{\begin{array}{l}
t \bar{t} \tilde{t}_{1} \tilde{t}_{1}^{*} \rightarrow t \bar{t} c \bar{c}+\mathbb{E}_{T} \\
t t \tilde{t}_{1}^{*} \tilde{t}_{1}^{*} \rightarrow t t \bar{c} \bar{c}+\mathbb{E}_{T} \\
\bar{t} \bar{t} \tilde{t}_{1} \tilde{t}_{1} \rightarrow \bar{t} \bar{t} c c+\mathbb{E}_{T}
\end{array}\right.
$$

This LHC signal has been studied in [70] using the like-charge lepton signal arising from the leptonic decay modes for both top quarks, with the result that both discovery and mass measurements are possible up to a gluino mass of about $900 \mathrm{GeV}$. The assumptions in the benchmark models used in that paper included a neutralino and stop that were both relatively lighter than in the scenario discussed in the present paper, but the results seem likely to be qualitatively the same.

Since many of the squarks produced at the LHC will decay through gluinos and then into top squarks and top quarks by eqs. (4.1), one expects also the same signal as in eq. (4.8) with two extra (usually light-flavor) jets, some of which may however be relatively soft. Other squark-squark and gluino-squark events will yield the typical jets plus leptons plus $\mathbb{E}_{T}$ signatures.

For models like the one in Figure 5, sleptons will not appear with significant multiplicity in the decays of the squarks and sleptons that are produced directly at the highest rates at the LHC. For these masses, the direct production of sleptons would also be very difficult to observe in either Drell-Yan production [71] or vector boson fusion [72].

If the decay mode $\tilde{t}_{1} \rightarrow W b \tilde{N}_{1}$ is open, as can occur for models with lower $\left|A_{0}\right|$ and/or higher $\Omega_{\mathrm{DM}} h^{2}$, then a different like-charge lepton signal results from $p p \rightarrow \tilde{g} \tilde{g} \rightarrow t t \bar{b} \bar{b} \ell^{-} \ell^{-}+\mathbb{E}_{T}$ and $\bar{t} \bar{t} b b \ell^{+} \ell^{+}+\mathbb{E}_{T}$ at the LHC. The resulting events with four potential $b$ tags, like-charge dileptons, and large missing energy should provide for a striking signal.

Finally, it is important to note that the scenario for neutralino LSP annihilation to a top quark does not present a particularly promising situation for a linear collider with $\sqrt{s}=500 \mathrm{GeV}$. In the model shown in Figure 5, and in a great deal of the allowed parameter space in Figure 2, the only supersymmetric particle that can be produced at such a machine is $\tilde{N}_{1}$, which does not lead to a visible signal (except possibly through initial state radiation $e^{+} e^{-} \rightarrow \tilde{N}_{1} \tilde{N}_{1} \gamma$ [73]). With that collision energy, only an $h^{0}$ with properties nearly indistinguishable from a Standard Model Higgs boson will be in direct evidence. Fortunately, if this is the course that Nature has chosen, the 
LHC should be able to identify the problem in advance, and allow for informed decisions regarding required beam energy. However, the difficulty in seeing sleptons at the LHC as noted above will present, as it does in many models, a challenge for assessing the capabilities of a linear collider.

\section{OUTLOOK}

In this paper, I have argued that there is a particularly appealing region of parameter space in which the right amount of dark matter can be obtained naturally, in the sense that $\Omega_{\mathrm{DM}} h^{2}$ varies rather slowly with changes in the input parameters. The key feature is suppression of $\Omega_{\mathrm{DM}} h^{2}$ due primarily to neutralino pair annihilation to top quarks through top squark exchange, which can occur with moderate top-squark mixing in models that have a relatively light gluino compared to the predictions of models with universal gaugino mass boundary conditions as in mSUGRA. The resulting superpartner spectrum can therefore be described as compressed, and leads to rather distinctive predictions. The fact that the $\tilde{N}_{1}$ mass has to exceed the top quark mass in this scenario for dark matter makes the discovery of supersymmetry impossible ${ }^{\dagger}$ at the past CERN LEP $e^{+} e^{-}$ collider, very difficult for the ongoing Tevatron, but quite promising for the LHC. Since the $\tilde{t}_{1}$ squark has to be still heavier by tens of $\mathrm{GeV}$, there is considerable doubt that a linear collider would be able to see it, or any other superpartners, unless the center-of-mass energy is higher than $\sqrt{s}=500 \mathrm{GeV}$.

In this paper, I have not attempted any detailed study of LHC potential for discovery or precision measurements, or of the possibilities for direct or indirect detection of the dark matter. These issues will hopefully be addressed in future work.

Acknowledgments: I am grateful to James Wells for useful conversations. This work was supported by the National Science Foundation under Grant No. PHY-0456635.

[1] S. Weinberg, Phys. Rev. D 13, 974 (1976), Phys. Rev. D 19, 1277 (1979); E. Gildener, Phys. Rev. D 14, 1667 (1976); L. Susskind, Phys. Rev. D 20, 2619 (1979); G. 't Hooft, in Recent developments in gauge theories, Proceedings of the NATO Advanced Summer Institute, Cargese 1979, (Plenum, 1980).

[2] For a review that uses the notations and conventions followed here, see S.P. Martin, "A supersymmetry primer," hep-ph/9709356 (version 4, June 2006).

[3] H. Goldberg, Phys. Rev. Lett. 50, 1419 (1983); J. Ellis, J. Hagelin, D.V. Nanopoulos, K. Olive, and M. Srednicki, Nucl. Phys. B 238, 453 (1984).

[4] M. Drees and M.M. Nojiri, Phys. Rev. D 47, 376 (1993) hep-ph/9207234.

[5] For reviews of different aspects of supersymmetric aspects of dark matter and relations with collider physics, see for example: G. Jungman, M. Kamionkowski and K. Griest Phys. Rept. 267, 195 (1996) hep-ph/9506380]; J.D. Wells, "Mass density of neutralino dark matter", hep-ph/9708285; K.A. Olive, "TASI Lectures on Dark matter," astro-ph/0301505]; J.L. Feng, "Supersymmetry and cosmology," hep-ph/0405215; M. Drees, "Neutralino dark matter in 2005," hep-ph/0509105, E.A. Baltz, M. Battaglia, M.E. Peskin and T. Wizansky, Phys. Rev. D 74, 103521 (2006) hep-ph/0602187].

[6] G. Belanger, F. Boudjema, A. Pukhov and A. Semenov, "micrOMEGAs2.0: A program to calculate the relic density of dark matter in a generic model," Comput. Phys. Commun. 176, 367 (2007)

\footnotetext{
$\dagger$ This is hardly a bold prediction now, but it does have the virtue of being inevitable in this scenario.
} 
hep-ph/0607059, Comput. Phys. Commun. 174, 577 (2006) hep-ph/0405253, Comput. Phys. Commun. 149, 103 (2002) hep-ph/0112278.

[7] P. Gondolo, J. Edsjö, P. Ullio, L. Bergstrom, M. Schelke and E.A. Baltz, "DarkSUSY: Computing supersymmetric dark matter properties numerically," JCAP 0407, 008 (2004) astro-ph/0406204;

[8] D.N. Spergel et al. [WMAP Collaboration], "Wilkinson Microwave Anisotropy Probe (WMAP) three year results: Implications for cosmology," astro-ph/0603449.

[9] M. Tegmark et al. [SDSS Collaboration], Phys. Rev. D 69, 103501 (2004) astro-ph/0310723.

[10] W.M. Yao et al. [Particle Data Group], "Review of particle physics," J. Phys. G 33, 1 (2006).

[11] R. Barate et al. [LEP Working Group for Higgs boson searches], Phys. Lett. B 565, 61 (2003) hep-ex/0306033. S. Schael et al. [LEP Working Group for Higgs boson searches], Eur. Phys. J. C 47, 547 (2006) hep-ex/0602042.

[12] E. Brubaker et al. [Tevatron Electroweak Working Group], "Combination of CDF and D0 results on the mass of the top quark," hep-ex/0608032.

[13] G.B. Gelmini and P. Gondolo, Phys. Rev. D 74, 023510 (2006) hep-ph/0602230, G. Gelmini, P. Gondolo, A. Soldatenko and C.E. Yaguna, Phys. Rev. D 74, 083514 (2006) hep-ph/0605016.

[14] A. Djouadi, M. Drees and J.L. Kneur, Phys. Lett. B 624, 60 (2005) hep-ph/0504090].

[15] J. Edsjö and P. Gondolo, Phys. Rev. D 56, 1879 (1997) hep-ph/9704361.

[16] J.L. Feng, K.T. Matchev and F. Wilczek, Phys. Lett. B 482, 388 (2000) hep-ph/0004043, K.L. Chan, U. Chattopadhyay and P. Nath, Phys. Rev. D 58, 096004 (1998) hep-ph/9710473, J.L. Feng, K.T. Matchev and T. Moroi, Phys. Rev. Lett. 84, 2322 (2000) hep-ph/9908309; Phys. Rev. D 61, 075005 (2000) hep-ph/9909334.

[17] K. Griest and D. Seckel, Phys. Rev. D 43, 3191 (1991).

[18] J.R. Ellis, T. Falk and K.A. Olive, Phys. Lett. B 444, 367 (1998) hep-ph/9810360; J.R. Ellis, T. Falk, K.A. Olive and M. Srednicki, Astropart. Phys. 13, 181 (2000) [Erratum-ibid. 15, 413 (2001)] hep-ph/9905481.

[19] M.E. Gomez, G. Lazarides and C. Pallis, Phys. Rev. D 61, 123512 (2000) hep-ph/9907261];

[20] C. Boehm, A. Djouadi and M. Drees, Phys. Rev. D 62, 035012 (2000) hep-ph/9911496;

[21] J.R. Ellis, K.A. Olive and Y. Santoso, Astropart. Phys. 18, 395 (2003) hep-ph/0112113;

[22] C. Balazs, M. Carena and C.E.M. Wagner, Phys. Rev. D 70, 015007 (2004) hep-ph/0403224.

[23] G. Belanger, F. Boudjema, S. Kraml, A. Pukhov and A. Semenov, Phys. Rev. D 73, 115007 (2006) hep-ph/0604150.

[24] A. Corsetti and P. Nath, Phys. Rev. D 64, 125010 (2001) hep-ph/0003186.

[25] A. Birkedal-Hansen and B.D. Nelson, Phys. Rev. D 64, 015008 (2001) hep-ph/0102075], Phys. Rev. D 67, 095006 (2003) hep-ph/0211071.

[26] U. Chattopadhyay, A. Corsetti and P. Nath, Phys. Rev. D 66, 035003 (2002) hep-ph/0201001.

[27] H. Baer, C. Balazs, A. Belyaev, R. Dermisek, A. Mafi and A. Mustafayev, JHEP 0205, 061 (2002) hep-ph/0204108.

[28] V. Bertin, E. Nezri and J. Orloff, JHEP 0302, 046 (2003) hep-ph/0210034.

[29] U. Chattopadhyay and D.P. Roy, Phys. Rev. D 68, 033010 (2003) hep-ph/0304108.

[30] D.G. Cerdeño and C. Muñoz, JHEP 0410, 015 (2004) hep-ph/0405057.

[31] G. Belanger, F. Boudjema, A. Cottrant, A. Pukhov and A. Semenov, Nucl. Phys. B 706, 411 (2005) hep-ph/0407218.

[32] G. Belanger, F. Boudjema, A. Cottrant, A. Pukhov and A. Semenov, Czech. J. Phys. 55, B205 (2005) hep-ph/0412309.

[33] Y. Mambrini and E. Nezri, hep-ph/0507263.

[34] S.F. King and J.P. Roberts, JHEP 0609, 036 (2006) hep-ph/0603095; JHEP 0701, 024 (2007) hep-ph/0608135.

[35] H. Baer, A. Mustafayev, E.K. Park, S. Profumo and X. Tata, JHEP 0604, 041 (2006) hep-ph/0603197, H. Baer, A. Mustafayev, S. Profumo and X. Tata, Phys. Rev. D 75, 035004 (2007) hep-ph/0610154.

[36] N. Arkani-Hamed, A. Delgado and G.F. Giudice, Nucl. Phys. B 741, 108 (2006) hep-ph/0601041].

[37] A. Falkowski, O. Lebedev and Y. Mambrini, JHEP 0511, 034 (2005) hep-ph/0507110. 
[38] H. Baer, E.K. Park, X. Tata and T.T. Wang, JHEP 0608, 041 (2006) hep-ph/0604253]; "Collider and Dark Matter Phenomenology of Models with Mirage Unification," hep-ph/0703024.

[39] K.J. Bae, R. Dermisek, H.D. Kim and I.W. Kim, "Mixed bino-wino-higgsino dark matter in gauge messenger models," hep-ph/0702041.

[40] V. Berezinsky et al., Astropart. Phys. 5, 1 (1996) hep-ph/9508249].

[41] P. Nath and R. Arnowitt, Phys. Rev. D 56, 2820 (1997) hep-ph/9701301.

[42] H. Baer, C. Balazs, M. Brhlik, P. Mercadante, X. Tata and Y. Wang, Phys. Rev. D 64, 015002 (2001) hep-ph/0102156.

[43] J.R. Ellis, T. Falk, K.A. Olive and Y. Santoso, Nucl. Phys. B 652, 259 (2003) hep-ph/0210205. J.R. Ellis, A. Ferstl, K.A. Olive and Y. Santoso, Phys. Rev. D 67, 123502 (2003) hep-ph/0302032. J.R. Ellis, K.A. Olive, Y. Santoso and V.C. Spanos, Phys. Lett. B 603, 51 (2004) hep-ph/0408118.

[44] H. Baer, A. Mustafayev, S. Profumo, A. Belyaev and X. Tata, Phys. Rev. D 71, 095008 (2005) hep-ph/0412059.

[45] A. De Roeck et al., "Supersymmetric benchmarks with non-universal scalar masses or gravitino dark matter," hep-ph/0508198.

[46] J.L. Evans, D.E. Morrissey and J.D. Wells, "Higgs boson exempt no-scale supersymmetry and its collider and cosmology implications," hep-ph/0611185.

[47] G.L. Kane and S.F. King, Phys. Lett. B 451, 113 (1999) hep-ph/9810374, M. Bastero-Gil, G.L. Kane and S.F. King, Phys. Lett. B 474, 103 (2000) hep-ph/9910506.

[48] B.C. Allanach et al., in Proc. of the APS/DPF/DPB Summer Study on the Future of Particle Physics (Snowmass 2001) ed. N. Graf, Eur. Phys. J. C 25113 (2002) hep-ph/0202233. J.A. Aguilar-Saavedra et al., Eur. Phys. J. C 46, 43 (2006) hep-ph/0511344.

[49] S.P. Martin and M.T. Vaughn, Phys. Rev. D 50, 2282 (1994) hep-ph/9311340, Y. Yamada, Phys. Rev. D 50, 3537 (1994) hep-ph/9401241, I. Jack and D.R.T. Jones, Phys. Lett. B 333, 372 (1994) hep-ph/9405233, I. Jack, D.R.T. Jones, S.P. Martin, M.T. Vaughn and Y. Yamada, Phys. Rev. D 50, R5481 (1994) [hep-ph/9407291].

[50] J.R. Ellis, K. Enqvist, D.V. Nanopoulos and K. Tamvakis, Phys. Lett. B 155, 381 (1985).

[51] M. Drees, Phys. Lett. B 158, 409 (1985).

[52] G. Anderson, C.H. Chen, J.F. Gunion, J.D. Lykken, T. Moroi and Y. Yamada, "Motivations for and implications of non-universal GUT-scale boundary conditions for soft SUSY-breaking parameters," report for Snowmass 96, hep-ph/9609457.

[53] G. Anderson, H. Baer, C. h. Chen and X. Tata, Phys. Rev. D 61, 095005 (2000) hep-ph/9903370.

[54] K. Huitu, Y. Kawamura, T. Kobayashi and K. Puolamaki, Phys. Lett. B 468, 111 (1999) hep-ph/9909227].

[55] K. Tobe and J.D. Wells, Phys. Lett. B 588, 99 (2004) hep-ph/0312159.

[56] S.P. Martin, "Predictions of the sign of mu from supersymmetry breaking models," Phys. Rev. D 65, 035003 (2002) hep-ph/0106280].

[57] A. Masiero, D. V. Nanopoulos, K. Tamvakis and T. Yanagida, Phys. Lett. B 115, 380 (1982), B. Grinstein, Nucl. Phys. B 206, 387 (1982).

[58] S.P. Martin and J.D. Wells, Phys. Rev. D 64, 036010 (2001) hep-ph/0011382.

[59] B.C. Allanach, "SOFTSUSY: A C++ program for calculating supersymmetric spectra," Comput. Phys. Commun. 143, 305 (2002) hep-ph/0104145.

[60] A. Djouadi, J.L. Kneur and G. Moultaka, "SuSpect: A Fortran code for the supersymmetric and Higgs particle spectrum in the MSSM," hep-ph/0211331].

[61] G.W. Bennett et al. [Muon g-2 Collaboration], Phys. Rev. D 73, 072003 (2006) hep-ex/0602035. Phys. Rev. Lett. 92, 161802 (2004) hep-ex/0401008. Phys. Rev. Lett. 89, 101804 (2002) [Erratum-ibid. 89, 129903 (2002)] [hep-ex/0208001]. Further discussion is found in the review of Höcker and Marciano in ref. [10].

[62] K.-i. Okumura and L. Roszkowski, Phys. Rev. Lett. 92, 161801 (2004) hep-ph/0208101].

[63] K.-i. Hikasa and M. Kobayashi, Phys. Rev. D 36, 724 (1987).

[64] The Planck collaboration, http://www.rssd.esa.int/Planck 
[65] F.E. Paige, S.D. Protopopescu, H. Baer and X. Tata, "ISAJET 7.69: A Monte Carlo event generator for p p, anti-p p, and e+ e- reactions," hep-ph/0312045.

[66] V.M. Abazov et al. [D0 Collaboration], "Search for the pair production of scalar top quarks in the acoplanar charm jet topology in $\mathrm{p}$ anti-p collisions at $\mathrm{s}^{* *}(1 / 2)=1.96-\mathrm{TeV}$," Phys. Lett. B 645, 119 (2007) hep-ex/0611003.

[67] [CDF Collaboration], "Search for Direct Pair Production of Scalar Top and Scalar Bottom Quarks in p-pbar Collisions at sqrt(s)=1.96 TeV", CDF note 8411, July 2006.

[68] R. Demina, J.D. Lykken, K.T. Matchev and A. Nomerotski, Phys. Rev. D 62, 035011 (2000) hep-ph/9910275.

[69] V. M. Abazov et al. [D0 Collaboration], Phys. Lett. B 638, 119 (2006) hep-ex/0604029, "Search for squarks and gluinos in events with jets and missing transverse energy with the $\mathrm{D} \emptyset$ detector using 1 $\mathrm{fb}^{-1}$ of RunIIa data", D $\emptyset$ Note 5312, January 2007.

[70] S. Kraml and A.R. Raklev, Phys. Rev. D 73, 075002 (2006) hep-ph/0512284.

[71] H. Baer, C.-h. Chen, F. Paige and X. Tata, Phys. Rev. D 49, 3283 (1994) hep-ph/9311248], S. Abdullin et al. [CMS Collaboration], J. Phys. G 28, 469 (2002) hep-ph/9806366.

[72] G. C. Cho, K. Hagiwara, J. Kanzaki, T. Plehn, D. Rainwater and T. Stelzer, Phys. Rev. D 73, 054002 (2006) hep-ph/0601063.

[73] S. Ambrosanio et al., Nucl. Phys. B 478, 46 (1996) hep-ph/9601292, A. Datta, A. Datta and S. Raychaudhuri, Eur. Phys. J. C 1, 375 (1998) hep-ph/9605432], G. Montagna, M. Moretti, O. Nicrosini and F. Piccinini, Nucl. Phys. B 541, 31 (1999) hep-ph/9807465, S.Y. Choi et al., Phys. Rev. D 60, 013007 (1999) hep-ph/9901368, H. Baer and A. Belyaev, "Associated neutralino neutralino photon production at NLC," in Proc. of the APS/DPF/DPB Summer Study on the Future of Particle Physics (Snowmass 2001) ed. N. Graf, hep-ph/0111017, H.K. Dreiner, O. Kittel and U. Langenfeld, Phys. Rev. D 74, 115010 (2006) [hep-ph/0610020]; "The Role of Beam polarization for Radiative Neutralino Production at the ILC," hep-ph/0703009. 